\title{
Research on the Organization of Digital Learning Resources
}

\author{
Zhiyin Yang \\ Information and Technology College, Jilin Agricultural University, Jilin, China \\ 23133124@qq.com
}

\begin{abstract}
Keywords: Digital Learning Resources; Organizational Methods; Organizational Model
\end{abstract}
\begin{abstract}
With the development of global network technology and digital information, the form of learning resources has changed greatly. Most of the learning resources are changed from traditional paper resources to digital learning resources. Scientific organization of learning resources is conducive to the effective use of digital learning resources. This paper analyzes the connotation, classification, the meaning, the method and the organization pattern of the digital learning resources, and analyzes the application of the organization method of digital learning resources in practice.
\end{abstract}

\section{Introduction}

Since the mid-1990s, Europe and the United States and other developed countries and regions, the digital information resources and digital learning resources, organization and construction of a high degree of attention. In recent years, China is also gradually pay attention to this part of the work.

Digital learning makes full use of modern information technology such as multimedia and network to provide a learning environment with rich resources and new communication mechanism. Through the effective integration of information technology and curriculum resources, the learners can realize the independent construction of learners' knowledge. Digital learning resources are the basic content and elements of digital learning, and the level of organization and application directly affects the effect of digital learning. At present, there are still many problems and shortcomings in the organization of digital learning resources.

\section{Overview of Digital Learning Resources}

Connotation of Digital Learning Resources. Learning resources refers to all the elements that can be used by the learner in the course of learning, mainly including the information of people and materials that support learning [1]. Digital learning resources refers to the digital processing based on the characteristics of learners to edit, you can run on a multimedia computer or network environment for learners to self-learning, and can achieve the sharing of multimedia materials [2]. It can inspire students through independent, cooperative, creative way to find and deal with information so that digital learning possible.

Classification of Digital Learning Resources. Digital learning resources according to the different ways can be divided into: digital video, digital audio, multimedia software, e-mail online learning management system, computer simulation online discussion data, file database and so on. Corresponding non-digital learning resources include: printing materials, slide film and video film and so on. Digital learning resources, some of the teaching environment-type resources, itself is not a pure resource, but it can rely on their own environment for learners to provide the corresponding learning resources services, based on this digital learning resources can be divided into resource-based learning resources and systems Environment - based learning resources.

\section{The Meaning of Digital Learning Resources Organization}

Conducive to Optimize the Quality of Digital Learning Resources. Digital learning resources are available in a wide range. With text, images, audio, video, software, database and other forms. Including a variety of disciplines, different areas, different regions, different languages of the various information. Therefore, the lack of quality and management mechanism to control the 
digital learning resources become more complicated, chaotic, and there are hidden dangers. These questions give the user a choice, using digital learning resources to bring obstacles. Only to its effective organization and construction in order to solve the digital resources varies greatly, chaos and other phenomena, so as to optimize the quality of digital learning resources.

Conducive to the Efficient Sharing of Digital Learning Resources. The Internet is an open network, although the digital learning resources can be shared on the Internet, but its distribution has a lot of freedom and randomness. As the organization and management of digital learning resources and there is no uniform standards and norms: the address of resources, links and content itself is often in the change, making the resource changes, extinction can not be predicted, making the digital learning resources have a strong disorder And instability, thus greatly reducing the sharing efficiency of digital learning resources. Under the effective organization, we can solve the problem of freedom and arbitrariness of its distribution, and increase its standardization and standardization. In the organization process, the important and meaningful resources organized in the database or a specific site, can be a good solution to the problem of resource changes in order to improve the efficiency of resource sharing.

Conducive to the Effective use of Digital Learning Resources. In the face of a large number of digital learning resources scattered in the network, learners need to learn a certain point of knowledge, had to go to the various subsystems to find the knowledge associated with the learning content, network courseware test questions. In the search process had to spend a lot of time, sometimes not necessarily be able to get all the knowledge related to learning resources. Only a strong organization of digital learning resources can improve the efficiency of the use of digital learning resources, so that each resource can be fully and effectively use. There will not be some digital learning resources have not been used or some resources are reused.

\section{The Organization Method of Digital Learning Resources}

Metadata methods. Metadata refers to the data used to describe the data characteristics and attributes, that is, data of the data [3]. The metadata organization method uses a number of general data units to describe the retrieval characteristics of the network information, describe the results or exist in the form of a database, or embedded in the information resources, the purpose is to make the network information resources management maintainers and users can Metadata to understand and identify resources [4]. The metadata is used to organize the data learning resources by using the DC metadata. It provides a simpler and more effective way for the description of the resources. It mainly describes the resource information of the Web resource by the digital resource provider, catalogs the resource contents and helps people as soon as possible in the discovery of the required digital learning resources.

Classification. The classification of various knowledge fields according to the principle of knowledge classification system, and to represent the number of categories, the letter symbol as the subject of the document logo, directly reflects the concept of knowledge classification system. This method objectively reflects the whole picture of knowledge and its intrinsic logical relationship. In the digital environment, the classification method to the use of databases, information kiosks, search engines and other tools on the binary digitization of the knowledge unit and learning resources to describe the stage [5]. Classification The organization is limited by the classification of disciplines and the scope of the search. The digital learning resources in accordance with the economics, law, education, literature, history, science, engineering, agriculture, medicine, military science, management, art and other disciplines first classification, and then each subject of digital learning resources According to the type of different types of resources, and finally the classification of resources after the organization logo, according to the logo will be stored in the digital learning resources organization.

Subject Method. The subject method is a method of organizing and revealing information in word order [6]. The subject of law. The organization of digital learning resources is mainly used to reveal the language in the language of the original language used by the author of the language or the author of the abstract. Including the words, free words and appear in the title of the document, 
abstract or text in the word, after the standard treatment directly as the theme of the document logo, according to the word order to organize and reveal information.

In the networked and digital environment, the subject organization method using the lexical relation chain to reveal the greatest advantage of the relevant knowledge can be fully demonstrated in the digital learning resources organization played a very significant role. There are two main types of subjective law: First, the use of existing vocabulary to organize digital learning resources. Its basic function is to promote better search results feedback and synchronic control through synonym control and the relationship between words to achieve higher accuracy, but in the digital learning resources organization, this method is not commonly used. Second, the key word method, keyword law in almost all search engines have been widely used. The website, the title, the address, the abstract, and the natural words in the text can be selected as keywords to create an index database. The user retrieves the hyperlink to the relevant digitized learning resource by retrieving the system's keyword search function.

\section{The Organization Model of Digital Learning Resources}

The Primary Organization Model of Digital Learning Resources. The primary organizational model of digital learning resources refers to the organization of the digital learning resources themselves. First of all, the network and learning in a variety of digital learning resources encountered in a large number of collection, after the collection of resources to evaluate the value and analysis. Retain valuable resources, remove valuable or meaningless digital learning resources. And then deal with the valuable value of the digital learning resources in accordance with the theme of the different content of the classification, and then each topic of the digital learning resources in accordance with the storage method of different re-classification. Finally, the resources are stored in different storage methods. Text class of digital learning resources can be set up to organize the database storage. Hypermedia or hypertext of the digital learning resources can use hypertext to organize storage. Some of the network of digital learning resources can use a dedicated web site for organizational storage.

Intermediate Learning Model of Digital Learning Resources. The intermediate organization model of digital learning resources refers to an organization model which is personalized to individual, disciplines, fixation and systematization. It is different from the first model of a comprehensive summary, but for a discipline or a specific user organization. The data collected by this organization model is selected and processed according to the requirements, and there is a certain depth of the order and control of the resources. According to this organizational model can be established subject information portal or personal digital learning resources library. This is a subjective organizational model based on user needs. Whether it is in the digital learning resources collection, collation, value analysis, or the final organization process has a large part of the subjective elements. Organize the learning resources according to the subjective needs of the user or the learner.

Advanced Organization Model of Digital Learning Resources. The advanced organization model of digital learning resources is to organize the resources of the organization again, and the concept of metadata is similar. This organizational model is a reorganization of the already organized digital learning resources, which will be based on the original organization, set up a composite organizational search tool and the establishment of the corresponding database, which contains the original digital learning resources of the organization information Key words, keywords or organization and organization mode, storage address, storage form and other information. When the learner needs to use the corresponding digital learning resources, the corresponding resources in the search information, the database can provide a number of information about the digital learning resources, and then according to the different needs of different digital learning resources. 


\section{Example analysis}

Resource-based digital learning resources learners use the most common and extensive. The following analysis of metadata in the use of resource-based digital learning resources in the application.

Resource-based digital learning resources and more types of information in the organization by hand is difficult to complete, so the need for computer processing. Metadata structure is simple, is the computer software can identify, and the establishment of the index for the user to retrieve the use of data [7], is the resource-based digital resource organization used by the resource description method. Metadata determines the organization and use of its resources by defining the organizational structure of its resources and the organizational structure of the resource library made up of digital objects. Currently used in the organization of the more commonly used metadata formats include: MARC format, DC metadata format.

MARC format is widely used in the description, storage, exchange, processing and retrieval of resource-based digital learning resources. But to the data age, too fine, professional and strong, demanding strict format difficult to cope with the rapid expansion of the amount of resources. At this time DC metadata came into being. The DC metadata is the metadata format for the minimum resource description. Usually used in the Web environment. It contains 15 elements: title, author, keyword, description, publisher, partner, type, format, identifier, source, language, association, coverage, etc. [8]. The entire set of elements is extensible and each element has repeatability and selectivity. DC metadata is widely used because of its flexibility, scalability, and compatibility.

In the organization of resource-based digital learning resources, the first thing is to use metadata to describe the contents and location of resource objects, DC metadata is the identification of resource objects. The use of different metadata systems, the metadata system required by the various metadata systems, through the establishment of mapping, translation and other methods into a metadata system. As the digital learning resources are not specific entities exist, it is important to make it clear. The exact address in the identifier element of the DC metadata contains information about the location of the resource-based digital learning resource, thereby determining the location of the resource, facilitating the discovery and retrieval of its resource objects, and spanning time and space constraints. The elements of the DC metadata during the retrieval process become the most important basis for users to search for resource-based digital resources. Dublin core elements: title, subject, creator, other contributors can be used as a search point, in addition, the contents of these elements can be optimized. The use of control vocabulary and regulatory control, to better play the function of the search. DC metadata records the basic characteristics of resource-based digital resources, the basic reflection of the profile and so on. To a certain extent, the user can be any element as a basis for selection, it can in some ways on behalf of resources, can support the choice of resources. In addition, during the organization, the DC metadata provides basic information about the name, content, age, format, and creator of the resource-based digital learning resource information object so that the user can view the resource object without having to browse the resource object itself With the basic understanding and understanding, with reference to the relevant standards can be the necessary assessment of its value, as a reference to access and use.

\section{Conclusion}

With the continuous application of computers and networks in the field of education, especially the continuous development of modern distance network education, teaching resources and learning resources in the form and content have been greatly changed. Digital learning is an increasingly important role in the play of our learning life. Digital learning resources are the key to digital learning, so the organization of digital learning is becoming more and more important. This paper focuses on the overview, connotation and characteristics of digital learning resources. Discusses the meaning of the organization of digital learning resources. This paper puts forward three methods of digital learning resource organization and digital learning resources. Finally, we use the organization method of digital learning resources to carry out case study. 


\section{References}

[1] Y.Y. Sun: Talent, (2009) No. 2, p.91. (In Chinese)

[2] Q.Z. Shi: Modern Educational Technology, Vol.17 (2007) No.2, p.30. (In Chinese)

[3] L.M. We: Modern Information, Vol. 25 (2005) No.4 p.76. (In Chinese)

[4] J.A .Younger: Library Trends, (1997) No. 3.

[5] L.T. Wang, H. Jia: Library Forum, Vol. 24 (2004) No. 2 p.22. (In Chinese)

[6] Y.F. Zhang, X.Y. Fu: University Library Work, (2008) No. 2 p.26. (In Chinese)

[7] Y.M. Chen: Journal of Library and Information Science, Vol. 21(2003) 40. (In Chinese)

[8] X.H. Geng: Modern Information, Vol. 24(2004) No. 7 p. 94. (In Chinese) 